\title{
Anxiety, phobia, and depression in patients with temporal arachnoid cyst-a case report
}

\begin{abstract}
Arachnoid cysts are the most common intracranial cysts and their role in causing psychiatric symptoms is often under recognized. Their clinical manifestations vary and are often unspecific. Among the most common complaints are headache, ataxia, seizures, dizziness, and visual changes. A few cases with associated psychiatric symptoms have been reported. Here we presented a 13-year-old right-handed female patient with normal development, presented with significant unspecified depressive, anxious, phobia and déjà vu, as well as somatic symptoms, including dizziness, vertigo, and headaches. She showed poor clinical response to Ability and valproic acid. She presented normal findings in neurologic and physical examinations as well as in the basic laboratory workups. However, the incidental head MRI showed a large arachnoid cyst involving the whole right temporal lobe.
\end{abstract}

In recent years special interest exhibited in whether intracranial arachnoid cysts are the source of psychological or psychiatric problems. Our case along with the literature reviewed made us to think arachnoid cysts seem to play an essential role in the disturbance in affection and cognition as well. It is highly suggested that the arachnoid cysts should be removed in some situations to relieve the refractory psychiatric symptoms.
Volume 10 Issue 5 - 2020

\author{
Chunhui Yang, ${ }^{1,4}$ Junyi Zhang,' Xiaoxue \\ Zhang,' Naiqi Feng, ${ }^{2}$ Limei Hao, ${ }^{3}$ Sandeep \\ Gaonkar ${ }^{4}$ \\ 'Beijing Ivheart Health Management Co., LTD, Department \\ of Neurology\& Neurosurgery, People's Hospital of Ordos \\ Dongsheng District, China \\ 2Department of Medical Ultrasonics, People's Hospital of Ordos \\ Dongsheng District, China \\ ${ }^{3}$ Beijing Emergency General Hospital, China \\ ${ }^{4}$ Conventions Psychiatry \& Counseling, USA
}

\begin{abstract}
Correspondence: Chunhui Yang, Beijing lvheart Health Management Co., Conventions Psychiatry \& Counseling, USA,
\end{abstract} Email chunhuiyang2018@gmail.com

Received: September 29, 2020 | Published: October I5, 2020

Keywords: arachnoid cyst, depressive disorder, temporal lobe, anxiety, phobia

\section{Introduction}

Arachnoid cysts accounts for around $1 \%$ of all intracranial spaceoccupying lesions and is a relatively common neurodevelopmental disorder with an estimated prevalence of $0.2 \%$ to $1.7 \% .^{1-3}$ The most common locations for intracranial arachnoid cysts are near the temporal lobe and there are genetic mechanisms behind its formation. ${ }^{1}$ In most cases, arachnoid cysts are present at birth (congenital) and may appear to be increasing in size. ${ }^{1-5}$ However, patients may live their entire life without any explicit symptoms from the cyst, even if the cyst is large. ${ }^{3-6}$ In the cases which symptoms occurred, their clinical manifestations differ and are often unspecific. When clinical symptoms are present, the most frequent manifestations are headache, dizziness, and convulsive episodes. ${ }^{1-4}$ But some articles have reported patients with intracranial arachnoid cysts presenting with psychiatric symptom. ${ }^{1-11}$

Here we would like to present a case in which a 13-year-old female with medium IQ lever has been developing psychological and psychiatric symptoms from childhood and the symptoms worsen with aging. An unintended head MRI revealed a large arachnoid cyst in the right temporal lobe which might explain the symptoms. There are reports of patients being cured of their psychiatric symptoms following neurosurgical intervention. It is highly suggested that arachnoid cysts may be removed to better the symptoms. ${ }^{1-11}$

\section{Case presentation}

A 13-year-old right-handed Asian female presented to our facility companied by her parents in August of 2020, for evaluation of the symptoms of depression, anxiety, phobia, and somatic symptoms for two years. The patient's mother stated that her right temporal prominence was noted at birth and it was thought that it might have been due to birth canal compression. As a toddler, she had a larger head circumference and showed that she could not fit into hoodies of her age. However, her physical and intellectual development was normal, and with her developing, the head circumference was not abnormal compared to children of her age.

Although her school score could reach B, she acknowledged she had to studied extremely hard and made a lot of effort to memorize the studied materials. She had no head trauma history.

She is quiet and socially awkward. Since she started elementary school in 2013, she has been excessively nervous and anxious before every test, even to the points of abdominal cramps. She had a low selfesteem and was always blaming herself. Sometimes when teachers criticized other students, she thought it is about her too. When she was in the fifth grade, she felt that there was no point in living, and often talked about wanting to be euthanized. But overall, she coped well with school life and was willing to get along with her classmates.

However, in 2019, when she moved into sixth grade, the symptoms began to worsen. She had complaints of markedly diminished pleasure, loss of energy and feelings of worthlessness. Whenever she came home from school, she had to stay in bed, feeling very tired on weekends too. She was reluctant to go out for social. It was hard for her to concentrate on the lectures. She felt forgetful. She had suicidal ideations but no attempts. She had somatic symptoms of chest tightness, breathlessness, abdominal pain, and diarrhea. She would bite her own arm or her parents' arm when she felt extremely anxious.

In early 2020, she began to experience dizziness, vertigo, and headaches presenting in the forehead or throughout the head. She had déjà vu, but no seizures.

In May of 2020, she was diagnosis as depressive disorder not otherwise specified and received medication (Lexapro+ Cid) for two months, but no significant therapeutic effect was seen. In July of 2020, she had nightmares almost every night that included the contents of suicide or something she killed. She felt terrified that she always felt 
like the room was full of ghosts during the day (not seen but felt). The thought of going to school made her very frightened and anxious. When she was extremely anxious, she was strongly in urge to touch her mother's body, especially to touch her breasts.

In August of 2020, she started using Abilify and valproic acid which made her symptoms improved in some degree, manifesting as suicidal thoughts and nightmares were disappearing, but intense anxiety and phobia remains.

In August, an unintended MRI of the head revealed a large arachnoid cyst involving the whole right temporal lobe (Figure 1). But no markable findings in neurologic and physical examinations. The results of basic laboratory workups, including $\mathrm{CBC}$, serum chemistry, TSH test, were all within normal limits.

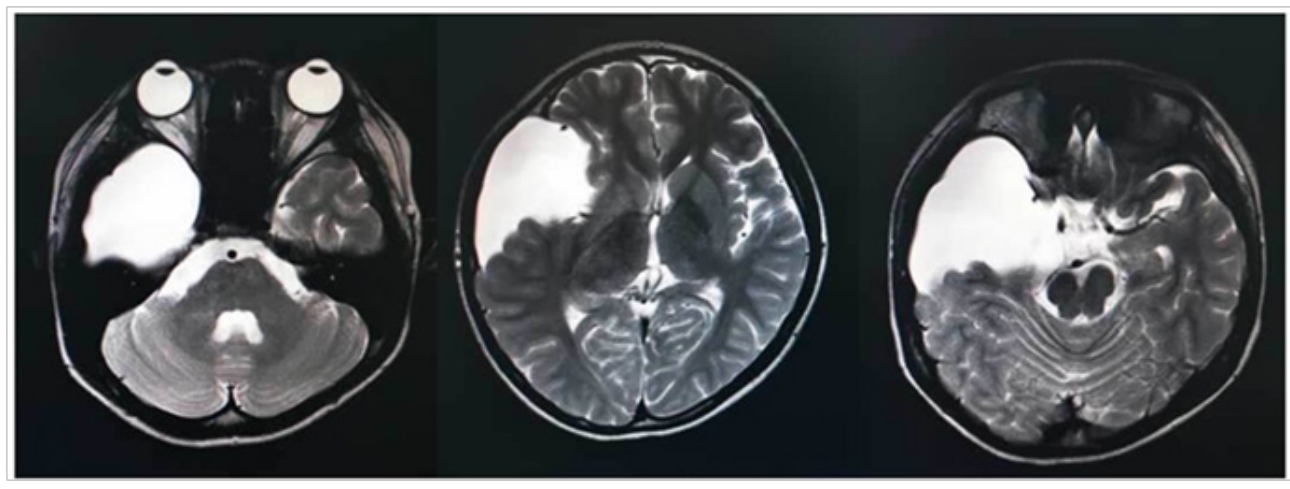

Figure I T2-weighted MRI showed an arachnoid cyst over the entire right temporal lobe.

\section{Discussion}

There is always a debate whether the cyst directly produces the psychiatric symptoms, ${ }^{1-11}$ or it is only an unintentional spectator. Despite our case lacking the development history of her cystic lesion, we would say the patient's abnormal behavior was likely secondary to the arachnoid cyst which was probably congenital in source because her right temporal prominence was noted at birth and she had no history of head trauma. Although physically and intellectually she had developed normally, she had mild psychological abnormalities in her childhood (easy to be nervous and social awkward) and significant psychiatric symptoms for over two year. And from the timeline of the symptoms, we conferred that the size of the lesion is increasing as she was developing which made her symptoms worsen. In addition, she only achieved mild and temporary stability with trials of antipsychotic and mood stabilizer in the outpatient settings. As above, we think our report discovered the possible causal relationship between an arachnoid cyst and psychiatric symptoms.

Many clinical reports suggested patients with intracranial arachnoid cysts may live their entire life without any apparent symptoms from the lesions. ${ }^{1-11}$ But recent literatures showed some interest in whether arachnoid cysts are the cause of psychological or psychiatric problems. ${ }^{1-11}$ Some studies found the patients with arachnoid cyst have higher levels of anxiety and depression compared with the general population. ${ }^{2}$ The suspected psychiatric disturbances secondary to arachnoid cysts could be of varied presentations like mood symptoms, schizophrenia-like psychosis, or amnestic symptoms. ${ }^{4-6}$

Studies indicated there was a significant difference in anxiety and depression scores between those with right-sided cysts and those with left-sided cysts. ${ }^{2}$ It has been identified that the right temporal lobe lesion more easily causes point-internal symptoms, such as anxiety and phobia, while left temporal lobe lesions would show pointexternal abnormalities, such as aggressive and anger. Our case was highly consistent with the clinical findings of right temporal lobe lesions.

The severity of the psychiatric symptoms may be related with the intra-cystic pressure that may compromise the function of the adjacent cortex and reduce perfusion and metabolism in surrounding cortical regions. ${ }^{11} \mathrm{~A}$ few clinical studies have examined cognition and psychiatric symptoms in a larger series of patients with cysts before and after surgical decompression. ${ }^{1-11}$ They found these changes are reversible after decompression of the cyst, presenting as cognitive and mental symptoms normalized after surgical cyst decompression. Some study even found this kind of changes may occur as early as 4 hours after surgery. ${ }^{2}$ Baquero, et $a .^{6}{ }^{6}$ found an arachnoid cyst as the provocative cause of psychopathological symptoms and such symptoms could be the only clinical manifestation of the cyst. The report mentioned the termination of symptoms by neurosurgical removal of the large arachnoid cyst. ${ }^{2}$

This case study pointed out it is critical for clinicians to distinguish secondary psychiatric symptoms from primary ones to form a correct treatment strategy. This study provided valuable information when considering the indications for surgical cyst decompression as well.

\section{Conflicts of interest}

The authors have no conflicts of interest to declare.

\section{Funding}

None.

\section{Acknowledgment}

None.

\section{References}

1. Kamal Patel, Ajayi Oluwadamilare, Kelly Melvin, et al. Psychiatric manifestation in setting of arachnoid cyst:a case series. ABC journal of Psychiatry. 2019;4(2):1-4

2. Priyanthi B Gjerde, Knut Wester, et al. Anxiety and depression in patients with intracranial arachnoid cyst-A prospective study. World Neurosurgery. 2019;132:e645-e653.

3. M Shettar, R Karkal, R Misra, et al. Arachnoid cyst causing depression and neuropsychiatric symptoms: A case report. East Asian Arch Psychiatry. 2018; 28(2):64-67. 
4. Soumitra Das, Arjun Kartha, Sumesh Thoppil Purushothaman, et al. Arachnoid Cyst and Psychosis: The Troublemaker or Innocent Bystander. Indian J Psychol Med. 2017;39(2):194-195.

5. Afaque H Khan and Syed E Ahmed. Arachnoid cyst and psychosis. Cureus. 2017;9(9):e1707.

6. G.A. Baquero, Patricio Molero, Jorge Pla-Vidal, et al. A Schizophrenialike psychotic disorder secondary to an arachnoid cyst remitted with neurosurgical treatment of the cyst. The Open Neuroimaging Journal. 2014;8(1):1-4.

7. Priyanthi B Gjerde, Marit Schmid, Asa Hammar, et al. Intracranial arachnoid cysts: impairment of higher cognitive functions and postoperative improvement. Journal of Neurodevelopmental Disorders. 2013;5(1):21
8. Yavuz Selvi, Adem Aydin, Lutfullah Besiroglu. Atypical Psychiatric Symptoms Associated with Left Temporal Lesions: Two Cases. Bulletin of Clinical Psychopharmacology. 2010; 20:329-333.

9. Chi-Hua Yu, Ying-Jie Peng, Shih-Cheng Liao, et al. An unusual approach to a patient with an arachnoid cyst and depression. Taiwanese Journal of Psychiatry (Taipei). 2010;24(2).

10. da Silva JA, Alves A, Talina M, et al. Arachnoid cyst in a patient with psychosis: case report. Ann Gen Psychiatry. 2007 6:16.

11. Antonis Vakis, Dimitris I Koutentakis, Dimitris Karabetsos, et al. Psychosis-like syndrome associated with intermittent intracranial hypertension caused by a large arachnoid cyst of the left temporal lobe. British Journal of Neurosurgery. 2006,20(3):156-159. 